\title{
El cuento como estrategia didáctica para la enseñanza de las teorías de la evolución de Lamarck y Darwin-Wallace a nivel medio superior
}

The story as a teaching strategy for teaching evolution theories of Lamarck and DarwinWallace superior to average

\author{
María Lorena Yoloxochitl Karla Quintino Salazar \\ Universidad Pedagógica Nacional \\ quintino28@hotmail.com
}

\section{Resumen}

El objetivo de la presente investigación fue analizar el cuento como una estrategia didáctica en el proceso de enseñanza-aprendizaje para la comprensión de las teorías de la evolución de Lamarck y Darwin- Wallace. La naturaleza de la investigación fue del tipo cuantitativo. El tratamiento de resultados fue realizado mediante un Análisis de $\mathrm{X}^{2}$ (chi cuadrado) en la frecuencia de las respuestas del pretest y del postest, en función de discriminar entre los grupos estudiados. Con base en la hipótesis alternativa se estableció que la narrativa como estrategia didáctica promueve el aprendizaje de las teorías de Lamarck y Darwin-Wallace.

Palabras clave: Cuento, estrategia de enseñanza, teorías de la evolución.

\section{Abstract}

The aim of this study was to analyze the story as a teaching strategy in the process of teaching and learning for understanding the evolution theories of Lamarck and Darwin-Wallace. The nature of the research was quantitative. Treatment of results was performed by X2 analysis (chi square) in the 
frequency responses of the pretest and posttest, based on discriminating between groups. Based on the alternative hypothesis that the narrative was established as a teaching strategy promotes learning theories of Lamarck and Darwin-Wallace.

Key words: Story, teaching strategy, theories of evolution.

Fecha recepción: Enero 2013

Fecha aceptación: Mayo 2013

\section{Introducción}

En el caso de la enseñanza de la biología uno de los temas donde ha habido un mayor interés por buscar el cambio conceptual es en la comprensión de la evolución biológica a través de la selección natural. Esto se debe a dos razones, por una parte el tema engloba toda la biología, y hoy en día no se concibe a esta ciencia desprovista de la idea del cambio evolutivo (Mayr, 1982); pero también a que la explicación darwiniana de la evolución provoca que el alumno traiga a colación preconcepciones propias, de su patrimonio cultural y motivadas por sus creencias (en ocasiones religiosas), pero sobre todo de origen contextual, donde hay gran coincidencia entre el pensamiento de los alumnos y el pensamiento científico pre-darwiniano. Por todo ello parece requerir un enfoque histórico, para su enseñanza (Ruiz, 1997).

La evolución y el modelo darwiniano de selección natural juegan un papel central en la biología, por lo que los estudiantes deberán adquirir una formación adecuada de estos conceptos, considerando que son la base que organiza el conocimiento biológico y dado su carácter unificador debería de ser unos de los primeros conceptos a revisar dentro de los programas, ya que les permite comprender la naturaleza y el procedimiento de la ciencia (Guillén, 1994).

Uno de los motivos principales para realizar esta investigación, en parte generada por observaciones dentro de la práctica docente, es la dificultad para enseñar los conocimientos sobre las teorías de la evolución, que forman parte del programa de Biología II del Colegio de Ciencias y Humanidades, donde se limita a presentar las concepciones de Lamarck y Darwin-Wallace, en donde se observa que los alumnos sólo memorizan algunas frases "relevantes" de las teorías, tomadas de los libros de texto, sin 
comprender que estos enunciados se desarrollaron dentro de un contexto histórico, con un acervo de posiciones, conocimientos y datos, no de forma aislada. La enseñanza de la biología debe estar enfocada no sólo al conocimiento disciplinario que debe manejar el alumno, sino que es necesario ofrecer elementos que los capaciten para aprender, construir y manejar el conocimiento, para que comprenda los conceptos y teorías de dicha especialidad, con el fin de que se concientice acerca de la repercusión social que el trabajo de los científicos ha jugado históricamente (Duit, 2006).

Surge entonces el interés de explorar los conceptos que tienen los estudiantes sobre estas teorías, y se propone el cuento para evaluar si ayuda a la adquisición del aprendizaje correcto de dichas teorías y puedan explicar los conceptos esenciales en que se basan.

\section{Objetivos e hipótesis}

Objetivo General:

Elaborar, presentar y evaluar al cuento como estrategia para promover el aprendizaje de las teorías de Lamarck y Darwin-Wallace, en la enseñanza a nivel medio superior.

Objetivos específicos:

a) Evaluar las ideas previas en cuanto a las teorías de Lamarck y Darwin-Wallace de alumnos y profesores de nivel medio superior.

b) Comparar las ideas previas de los profesores que imparten este tema y los alumnos de nivel medio superior.

c) Evaluar los conceptos adquiridos después de la intervención.

La hipótesis central del presente trabajo fue que:

- El cuento promueve el aprendizaje del tema de las teorías de Lamarck y Darwin-Wallace.

\section{Marco teórico}

Los conocimientos transmitidos durante la educación deben ser mostrados como las soluciones logradas por los hombres en el curso de su enfrentamiento a los problemas prácticos y concretos de la vida real. Duit (2006), comenta, que la enseñanza de las ciencias, entre ellas la Biología, es una actividad interdisciplinaria, en las que son necesarias las competencias que proporcionan otras ciencias, abordándola desde diferentes enfoques.

Los grandes cambios que el mundo ha sufrido en las últimas dos décadas, han conducido al cuestionamiento de ciertos elementos que han estado presentes en el análisis de los fenómenos 
sociales y políticos. El problema de la transformación de la enseñanza en nuestro país se ha orientado fundamentalmente a los aspectos metodológicos. Un aspecto relevante, consistía en confrontar la enseñanza memorística, la cual era la norma, la repetición de textos o de apuntes aportados por el profesor derivada de una concepción autoritaria, fue totalmente cuestionada y se promovió la reflexión del trabajo académico (Suarez y López-Guazo, 1993).

Los profesionistas egresados de los diversos campos de la ciencia, generalmente poseen deficiencias en aspectos fundamentales de las bases filosóficas, metodológicas y epistemológicas vinculados con la disciplina; esta falta repercute no sólo en la forma cómo enseña su disciplina, sino en cómo lleva su trabajo cotidiano de investigación. Un manejo adecuado de los fundamentos epistemológicos de la biología y de la ciencia en general, repercutirá en que a los alumnos se les presente una visión realista del quehacer científico. Propiciar en ellos la comprensión de la temporalidad de la verdad científica, su constante transformación, para evitar esquemas dogmáticos (Suárez y López-Guazo, 1993).

La enseñanza constructivista basada en las ideas de Vigotsky y Piaget, postula que la enseñanza escolar de las ciencias inicia con la propia construcción que los estudiantes hacen de la realidad, de manera que para que los maestros conozcan de dónde partir en la enseñanza, deben buscar que los alumnos expresen sus propias ideas y las puedan aplicar en diversas situaciones; lo que evitaría, de acuerdo con el constructivismo, la tendencia de los maestros de ciencias a intentar imponer verdades (Sánchez, 2000).

Es por ello, que actualmente se sugiere integrar más directamente las teorías científicas y la historia de su desarrollo a la instrucción en ciencias en todos los niveles educativos, incluyendo la formación de maestros. Es importante iniciar la enseñanza de la ciencia, y en particular, de la teoría de la evolución, señalando que la ciencia busca la organización sistemática del conocimiento acerca del mundo, se interesa por fórmulas, leyes generales y teoría que relacionan diferentes fenómenos y procura explicar los suceso observables. También es posible entenderla como el conjunto de explicaciones que se han dado de los fenómenos naturales y de los métodos seguidos para llegar a tales explicaciones. El conocimiento científico surge por consenso entre los científicos, a partir del conocimiento previo y aunque no siempre concuerdan en algunas cuestiones no establecidas, suelen coincidir con el conocimiento ya establecido (Sánchez, 2000). 
De acuerdo con Hernández (2006), se puede afirmar que la enseñanza de la ciencia constituye un problema complejo, en el cual se observan dos tendencias:

a) La ciencia presentada como un cúmulo de resultados pero a históricos;

b) La ciencia tratada como algo que puede ser captada por los ejemplos de trabajo expuestos en los libros científicos, o lo que es más común, con ejemplos de la vida cotidiana.

\section{El conocimiento previo sobre la teoría de la evolución.}

La teoría evolutiva constituye el paradigma central de la biología moderna (Ruse, 1979). La importancia de esta teoría en el desarrollo conceptual de la biología ha sido enorme y, pese a las insuficiencias que todavía posee, se puede afirmar, como decía T. Dobzhansky, que "nada tiene sentido en biología, excepto bajo el prisma de la evolución". En efecto, la comprensión de la vida está ligada directamente a la concepción evolucionista (Castro, 2008).

La posibilidad de que surjan interpretaciones erróneas de las hipótesis científicas, o de que se produzca un mal uso de las mismas se incrementa a causa de un conocimiento insuficiente de los temas y a la confusión, más o menos interesada, de las cuestiones científicas con las cuestiones éticas y sociales. Para ello nada mejor que proporcionar a los alumnos una formación que les permita integrar, desde una perspectiva evolutiva los conocimientos biológicos que van a adquirir durante sus estudios y que, al tiempo, les sirva de soporte para una reflexión de carácter más profundo sobre el ser humano y su naturaleza (Castro, 2008).

La teoría de la evolución es un constructo unificador que permite dar continuidad al pasado y presente de la historia biológica, pero cuya complejidad presupone que, para comprenderla, el alumno posea pensamiento formal en el sentido piagetiano. Sin embargo, este tema que debería enseñarse mediante numerosos ejemplos y demostraciones, está en realidad pobremente explicado en los libros de texto, e impartido por maestros que suelen desconocer el nivel de conocimientos de sus estudiantes (Sánchez, 2000).

Es un hecho que los biólogos y los educadores de esta materia saben que aprender adecuadamente el proceso de evolución es crucial para que los alumnos comprendan e integren los procesos de la vida. En la práctica, sin embargo, no se logra la enseñanza de este tema por un sinfín de razones. Entre ellas están la actitud, el entorno y la capacidad cognoscitiva de los estudiantes; la complejidad de la propia teoría y las dificultades que tienen los maestros para abordarla. Pero en general las diferentes 
metodologías que se han propuesto para lograr que el alumno se apropie del conocimiento evolutivo, arrojan resultados no satisfactorios (Lawson y Worsnop, 1992).

Entre los factores que influyen en como una persona responde al enfrentarse a datos nuevos, están las características de su conocimiento previo; en el caso de la teoría de la evolución, están involucradas las creencias originales, en ocasiones religiosas, que son muy difíciles de cambiar (Chinn y Brewer, 1993). Por otro lado, para que los datos nuevos sean aceptables, se requiere de demostraciones, experimentos o vivencias que hagan referencia al mundo real o que sean perceptualmente obvias, lo cual resulta muy complicado para el caso particular de la evolución biológica. La dificultad en el aprendizaje de esta teoría ha determinado que en educación, exista una preocupación por entender cómo conciben los alumnos los mecanismos de la evolución, dado que sus ideas difieren de las sustentadas por los biólogos, en principio se considera que se trata de un enfrentamiento entre un concepto abstracto como lo es la teoría de la evolución y un concepto difícil de percibir como la variación, frente al pensamiento concreto de los estudiantes (Sánchez, 2000).

Existen varios problemas importantes a la hora del aprendizaje de la teoría evolutiva, una primera dificultad procede de la presencia en los alumnos y a veces en los maestros de una preconcepción lamarckista del proceso evolutivo (Bishop y Anderson, 1990; Jiménez-Aleixandre, 1992). En efecto, la idea que los cambios en los organismos se producen como respuesta a las condiciones del medio y que dichos cambios son heredables está fuertemente arraigada y no es sencillo conseguir un cambio conceptual hacia posiciones darwinistas. La lógica intuitiva inherente a las ideas lamarckistas y el hecho de que no exista un auténtico conflicto entre ambas teorías parecen ser las responsables de esta dificultad para adquirir un concepto clave en la teoría evolutiva. Una segunda cuestión, que también plantea dificultades importantes, radica en la identificación errónea del concepto de selección natural con la idea de la lucha por la existencia y del triunfo de los más fuertes, conceptos previos al darwinismo desarrollados por Herber-Spencer. Este planteamiento ha originado históricamente la utilización incorrecta de las ideas darwinistas como las expuestas por Marx (darwinismo social), que han servido de instrumento para justificar la estructura socioeconómica de las sociedades humanas, eugenesia y racismo. Por último, existen dificultades que provienen tanto de la propia complejidad de algunos conceptos teóricos, como el debate científico que existe en la actualidad sobre determinados aspectos del proceso evolutivo, lo que en conjunto dificulta una buena comprensión de la teoría 
evolutiva, por parte, no ya de los alumnos, sino de los propios docentes no expertos en este campo (Castro, 2008).

Cuando se revisan los diferentes textos relativos al estudio de la teoría de la evolución biológica, destaca la coincidencia respecto de la forma como se les debe enseñar este importante tema a los estudiantes. Inicia con un recorrido histórico del desarrollo del pensamiento humano, catastrofismofijismo, hasta concluir con las ideas evolucionistas no fijistas. Durante este recorrido, se pretende que los alumnos comprendan la importancia de estudiar desde esta óptica los procesos biológicos, como procesos completamente dinámicos y en constante cambio, aunque lo ideal sería que el pensamiento evolucionista se enseñara como una serie de ideas que han cambiado con el tiempo, comenzando con el pensamiento de Lamarck, continuando con el de Darwin-Wallace, y concluyendo con la teoría sintética y las posiciones neodarwinistas contemporáneas. El docente normalmente elige entre dos estrategias didácticas posibles: una simple explicación enumerativa de los ejemplos clásicos sobre evolución, o bien intentar acercar la teoría de la evolución al alumno, convertirla en algo real y observable. Considerando que este esquema es el ideal, se esperaría que los estudiantes adquirieran una serie de conocimientos adecuados acerca de la teoría de la evolución, sin embargo, la comprensión del tema y su visión sobre la evolución lleva a cuestionar seriamente el esquema vigente de enseñanza (Gersenowies, 2010).

Cuando se intercambian opiniones sobre el tema en ambientes sociales y académicos, no es raro constatar que personas con una preparación universitaria sigan afirmando que "Darwin dijo que descendíamos del mono", "La teoría de la evolución es de Darwin”, "El ejemplo de las jirafas es de Darwin", "La teoría de la evolución es eso, sólo una teoría", y muchas otras ideas equívocas que no sólo reflejan una completa incomprensión del desarrollo histórico de las ideas evolucionistas, sino de los principios metodológicos propios de la ciencia y de la estructura de un programa de investigación, que ha sido muy fructífero en los últimos 150 años, que es el estudio de la evolución biológica. Este estudio no está sujeto a demostrar de forma fehaciente que la teorías de Darwin o la teoría sintética son absolutas, sino todo lo contrario, se consideran provisionales y perfectibles, existiendo una serie de hechos que deben ser explicados dentro de una estructura lógica (Gersenowies, 2010). 
Existen una serie de errores con respecto a algunos temas que se discuten dentro de la enseñanza de la evolución, lo cual puede llegar a constituir fuentes de confusión, de los cuales se mencionan sólo los que tiene que ver con Lamarck y Darwin:

- Se considera que Darwin presentó su teoría de forma terminada, hecho que es falso.

- Se presenta la teoría de la evolución biológica como una explicación completamente terminada y no como un programa de investigación en desarrollo.

- Rara vez se menciona cuáles son los hechos que debe explicar la teoría, cuáles ha explicado y cuáles están en controversia.

- Se presentan muchos mitos en torno a la figura de Darwin y se reduce la contribución de investigadores como fueron Wallace, Fisher, Mayr, Stebbins y otros, hecho que desvirtúa la contribución real de Darwin.

- Uno de los ejemplos más frecuentes para explicar la teoría de la evolución es la comparación entre la evolución lamarkiana y la darwiniana utilizando el alargamiento del cuello de las jirafas, que aparece frecuentemente en los libros de texto. En la primera mitad del siglo XIX, Lamarck atribuyó el alargamiento del cuello a la herencia de modificaciones corporales causadas por el hábito de estirar el cuello. Darwin atribuyó el alargamiento del cuello a la selección constante de individuos y razas que nacieron con los cuellos más largos.

- Otro error clásico consiste en afirmar que Darwin utilizó como ejemplo de evolución a los pinzones, cuando sólo los menciona esporádicamente en su obra (Gersenowies, 2010).

\section{Antecedentes}

En la actualidad el docente cuenta con muchas formas de presentar su clase, utilizando diversos materiales y variadas estrategias, sin embargo son pocos los trabajos realizados acerca del uso del cuento, como recurso didáctico en la enseñanza de la biología, sobre todo en el nivel medio superior, pero existe un número considerable de trabajos realizados sobre la narrativa y su función educativa.

Así, por ejemplo, se puede mencionar a: Solbes y Traver (2001), quienes se centraron en el análisis crítico de la situación de la enseñanza habitual, respecto a la falta de una perspectiva histórica adecuada que ponga en evidencia la imagen deficiente de la naturaleza y evolución de la ciencia que se transmite a los alumnos y su influencia en el desinterés de los mismos hacia el aprendizaje de la física y la química. Los autores elaboraron materiales didácticos para impartir física y química con un enfoque 
histórico, basándose principalmente en la lectura de textos, biografías contextualizadas. Una vez utilizados los materiales, elaborados por los autores, se compararon los resultados obtenidos por los grupos experimentales y los grupos control, encontrando que los alumnos que han seguido un tratamiento histórico, muestran una imagen de la ciencia más contextualizada y próxima a la realidad, y en la mayoría se constata una disminución significativa de los que perciben la actividad científica como descubrimiento disminuye la visión acumulativa de la ciencia, mejora el conocimiento de los científicos y sus trabajos y quieren conocer el proceso de creación de la ciencia. En los grupos experimentales se detecta una mejor comprensión de los aspectos relacionados con la contextualización de los conocimientos científicos, también aumentan los alumnos que conocen los problemas generadores de diversos trabajos científicos. Finalmente, los autores concluyen que la falta de interés de los alumnos y su actitud caracterizada por la escasa apreciación hacia el estudio de las ciencias estaba motivada, en parte, por la visión ahistórica de la enseñanza impartida habitualmente, que muestra una imagen sesgada de la naturaleza de la ciencia y su evolución. Comprobaron, que es posible aumentar el interés hacia el estudio de la física y la química, mediante un tratamiento mínimamente detenido de algunos aspectos históricos, introducidos en el proceso de adquisición de los diferentes conceptos y teorías científicas, ya que se puede mostrar una imagen de la ciencia más correcta y próxima a la realidad del trabajo de los científicos y al contexto en que éste se desarrolla y se ha desarrollado a lo largo de la historia.

Singer (2006), investigó el efecto del cuento como estrategia de enseñanza-aprendizaje sobre el rendimiento estudiantil de los alumnos de secundaria en Venezuela, al abordar el tópico correspondiente a las propiedades de los compuestos químicos inorgánicos. El diseño corresponde a una investigación de campo, puesto que los datos son recabados en la realidad de los ambientes de aprendizaje. La hipótesis es que la estrategia de enseñanza y aprendizaje utilizando el cuento dramatizado debería favorecer un mayor rendimiento estudiantil que el uso del cuento ilustrado y, a su vez, el cuento sin ilustración debería corresponder al menor promedio de calificaciones en una prueba de rendimiento estudiantil de los contenidos estudiados. El tratamiento de los resultados fue realizado mediante un análisis de varianza de los promedios de las calificaciones y luego se aplicó la prueba de Tukey, en función de discriminar entre los grupos estudiados. La hipótesis del mayor rendimiento estudiantil como efecto de la administración del cuento dramatizado resultó confirmada por las 
pruebas estadísticas correspondientes; sin embargo, el cuento sin ilustración predominó sobre el cuento ilustrado, lo cual se explicaría por la libertad de imaginación y motivación en ausencia de ilustraciones.

Kalkanis y colaboradores (2010), realizaron su investigación con el objetivo de analizar la influencia de los cuentos en el proceso de enseñanza-aprendizaje para la comprensión y aplicación de la tabla periódica, en la asignatura de química del noveno grado de educación básica en Venezuela, que es el equivalente al tercer grado de secundaria en México. Su objetivo fue actualizar e incluir otros recursos didácticos en la enseñanza de la química; específicamente en la enseñanza de la tabla periódica, a través del uso del cuento como recurso didáctico muy sencillo; afirman que al escuchar o leer un cuento tiene muchos beneficios como son: desarrollar la habilidad de escucha e interacción, estimular a la lectura, aumentar el vocabulario y la capacidad de comprensión. Se preguntan ¿De qué manera puede influir el cuento como recurso didáctico en el proceso de enseñanza aprendizaje para la comprensión y aplicación de la tabla periódica? Para responder los autores determinaron el nivel de conocimiento de los estudiantes sobre el este tema, antes de aplicar el cuento como recurso didáctico. Los cuentos que fueron empleados, son producción propia de los autores. El diseño fue de pretestpostest y un grupo control, para estudiar el efecto de la aplicación de estrategias metodológicas de aprendizaje significativo. Los resultados obtenidos fueron analizados mediante la prueba de " $\mathrm{t}$ " de Students de los promedios de las calificaciones del pre-test y del postest, en función de discriminar entre los grupos estudiados. De los resultados obtenidos en esta investigación, se concluyó que el cuento como recurso didáctico es efectivo para la comprensión y aplicación de la tabla periódica.

\section{Método}

Con el objeto de desarrollar los objetivos planteados, se utilizó el siguiente método:

a) Grupos y sus criterios de elección: Los grupos de trabajo. fueron seleccionados de acuerdo a la disposición del Colegio de Ciencias y Humanidades, plantel Azcapotzalco, del ciclo 2009-2, turno vespertino; una de las características principales es que estuvieran cursando Biología II, de acuerdo con el programa del colegio, dos grupos control y dos grupos con experimentales elegidos al azar.

b) Diseño de cuestionario: Se elaboraron 20 items de opción múltiple, para explicar los conceptos sobre las teorías de Lamarck y Darwin- Wallace, a cada pregunta se le asignaron cuatro posibles respuestas breves, en donde sólo una es correcta. Estas preguntas se dividieron en dos cuestionarios balanceados 
de igual dificultad y contenidos, esto con el fin de evitar la memoria a largo plazo. Para la estructuración de las preguntas, se acotaron los conceptos claves de las teorías de Lamarck, como la herencia del los caracteres adquiridos y la escala natural. Los conceptos clave para la teoría de DarwinWallace, fueron el origen común y la selección natural.

c) De forma conjunta, para todos los grupos, se aplicó el cuestionario utilizado por Sánchez (2000), para conocer las ideas previas sobre el tema de evolución, a los cuatro grupos y para comparar con nuestros propios resultados.

d) Diseño de cuento.- Para la elaboración de la narración se seguirán los elementos sugeridos por Zavala (1994).

e) Análisis de los datos: se aplicaron las siguientes pruebas

-Se elaboraron tablas de frecuencias de respuestas lamarkianas y darwinianas entre los resultados de las preconcepciones de los alumnos ambos grupos y las preconcepciones de ambos grupos y los profesores.

-Se elaboraron tablas de frecuencias de respuesta correcta-incorrecta del pretest de los grupos control, experimental y profesores.

-Se elaboraron tablas de frecuencias de respuesta correcta-incorrecta del pretest y postest en el grupo control y experimental.

-Análisis de $\mathrm{X}^{2}$ de las tablas de frecuencia, para ello se utilizó el programa Statistica versión 7.

\section{Análisis de resultados}

Preconcepciones en los grupos control y experimental.

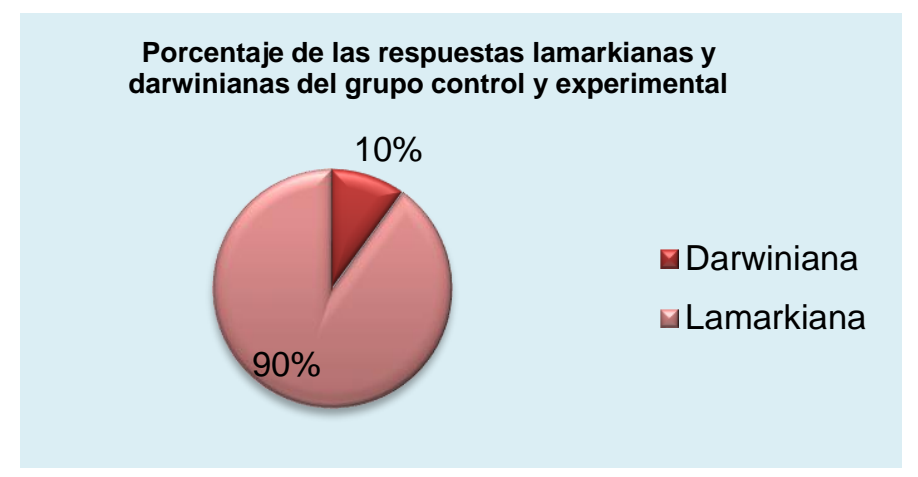

Los resultados muestran que el $\mathbf{9 0 \%}$ de las respuestas comparten preconcepciones lamarkianas para ambos grupos. Los alumnos tienen la idea de que los organismos cambian por necesidad para adaptarse al medio, estos cambios en los organismos se producen como respuesta a las condiciones 
del ambiente y que dichos cambios son heredables. Esta idea está fuertemente arraigada y no es sencillo conseguir un cambio conceptual hacia posiciones darwinianas. Sánchez (2000), Bishop y Anderson, (1990), y Jiménez-Aleixandre (1992), afirman que existen varios problemas al momento del aprendizaje de la teoría evolutiva, entre ellos están la actitud y la capacidad cognoscitiva del alumno, la complejidad de la propia teoría y las dificultades que tienen los maestros para abordarla. Otra posible explicación del porque la mayor parte de los alumnos conservan ideas lamarkistas es la que menciona Chinn y Brewer (1993), en cómo se presentan los nuevos datos y las características del conocimiento previo que poseen los aprendices además de la resistencia al cambio conceptual

Sánchez (2000) menciona que para que los datos nuevos sean aceptables, se requiere de demostraciones y experiencias vivenciales que para el caso de la teoría evolutiva es muy complicado por lo que se considera como un factor importante en la explicación de estos resultados, el papel que los docentes tienen en el proceso de enseñanza de la teoría evolutiva y los problemas de la didáctica de las ciencias son un obstáculo para el aprendizaje no solo de los alumnos sino también de los docentes (Campanario,1998).

\section{Comparación de las preconcepciones entre alumnos y profesores.}

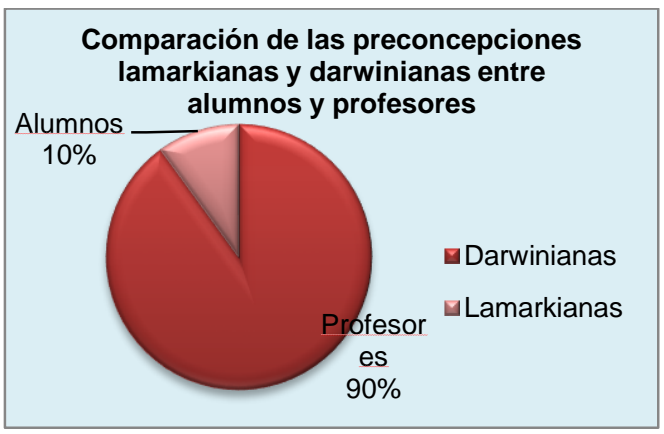

Se observó que en el $90 \%$ de las respuestas de la comparación entre las preconcepciones de los profesores con respecto a la de los alumnos, independientemente de la intervención cumple con lo esperado, ya que se parte de la premisa de que el maestro es el experto en las teorías evolutivas. 
Sin embargo se observa que en las respuestas incorrectas obtenidas de los profesores el $50 \%$ de los maestros comparten ideas lamarkianas con los alumnos en el sentido de que los organismos cambian por necesidad, para adaptarse al medio, estos cambios en los organismos se producen como respuesta a las condiciones del medio y que dichos cambios son graduales y heredables, lo cual explica el resultado obtenido en los alumnos y corrobora lo explicado por Campanario (1999), quienes mencionan que los maestros siguen teniendo modelos de enseñanza por transmisión por lo tanto el alumno sabe lo que el maestro le enseña.

Pretest de los alumnos del grupo control y experimental.

Pretest de los alumnos del grupo control y experimental.

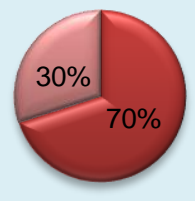

$$
\begin{aligned}
& \square \text { Similitud en } \\
& \text { conceptos } \\
& \square \text { Diferencias } \\
& \text { concptuales }
\end{aligned}
$$

En este punto se pudo observar que el $70 \%$ de los alumnos de los dos grupos presentan los mismos conceptos. Sin embargo se observa que el $30 \%$ de las respuestas en el grupo experimental mostro un mejor dominio de los conceptos de cambio gradual, escala natural y la herencia de los caracteres adquiridos, conceptos de la teoría de Lamarck, lo que concuerda con las preconcepciones observadas en donde predominas las lamarkianas.

Pretest entre los grupos control, experimental y los profesores

\begin{tabular}{|l|l|l|}
\hline & Alumnos & \multicolumn{2}{|l|}{$\begin{array}{l}\text { Profesores } \\
\text { Conceptos }\end{array}$} & $\begin{array}{l}\% \\
\text { respuestas } \\
\text { correctas }\end{array}$ & $\begin{array}{l}\text { despuestas } \\
\text { correctas }\end{array}$ \\
\hline Cambio gradual & $23 \%$ & $75 \%$ \\
\hline Polémica sobre la & & \\
\hline descendencia & $0 \%$ & $25 \%$ \\
\hline
\end{tabular}




\begin{tabular}{|l|l|l|}
\hline atribuida a Darwin & & \\
\hline $\begin{array}{l}\text { Autor del libro "El } \\
\text { origen de las }\end{array}$ & $72 \%$ & $100 \%$ \\
\hline especies" & & \\
\hline Selección natural & $44 \%$ & $100 \%$ \\
\hline
\end{tabular}

La comparación de los resultados en este punto confirma la tendencia del dominio de la teoría de Lamarck en la población estudiada. Sin embargo, llama la atención como para el concepto de cambio gradual que pertenece a la teoría de Lamarck el 77\% de los alumnos respondió equivocadamente, pero los profesores lo hicieron al $\mathbf{1 0 0 \%}$. De igual forma para el dato histórico de la polémica de la descendencia del mono, atribuida erróneamente a Darwin, sólo el $25 \%$ de los profesores respondieron correctamente, un error muy común mencionado por Gersenowies (2010).

Estos resultados se pueden explicar por lo mencionado por Suarez y López-Guazo (1993); los profesionistas de diversos campos de la ciencia generalmente poseen deficiencias en aspectos fundamentales de las bases filosóficas, metodológicas y epistemológicas vinculados con su disciplina. Díaz-Barriga y Hernández (2006), plantea que un docente debe tener presente al momento de planificar estrategias, entre otros aspectos el dominio del conocimiento científico en general y del contenido curricular en particular de la disciplina que pretende enseñar. Se debe aclarar que los maestros entrevistados son biólogos de profesión pero la mayoría no cuenta con una formación docente que le permita el diseño de estrategias de enseñanza.

Comparación entre el pretest y postest en el grupo control.

\begin{tabular}{|c|c|c|}
\hline Conceptos & $\begin{array}{l}\text { Pretest } \\
\% \\
\text { respuestas de } \\
\text { correctas }\end{array}$ & $\begin{array}{l}\text { Postest } \\
\% \\
\text { respuestas de } \\
\text { correctas }\end{array}$ \\
\hline $\begin{array}{l}\text { Herencia de los } \\
\text { caracteres } \\
\text { adquiridos }\end{array}$ & $80 \%$ & $100 \%$ \\
\hline
\end{tabular}




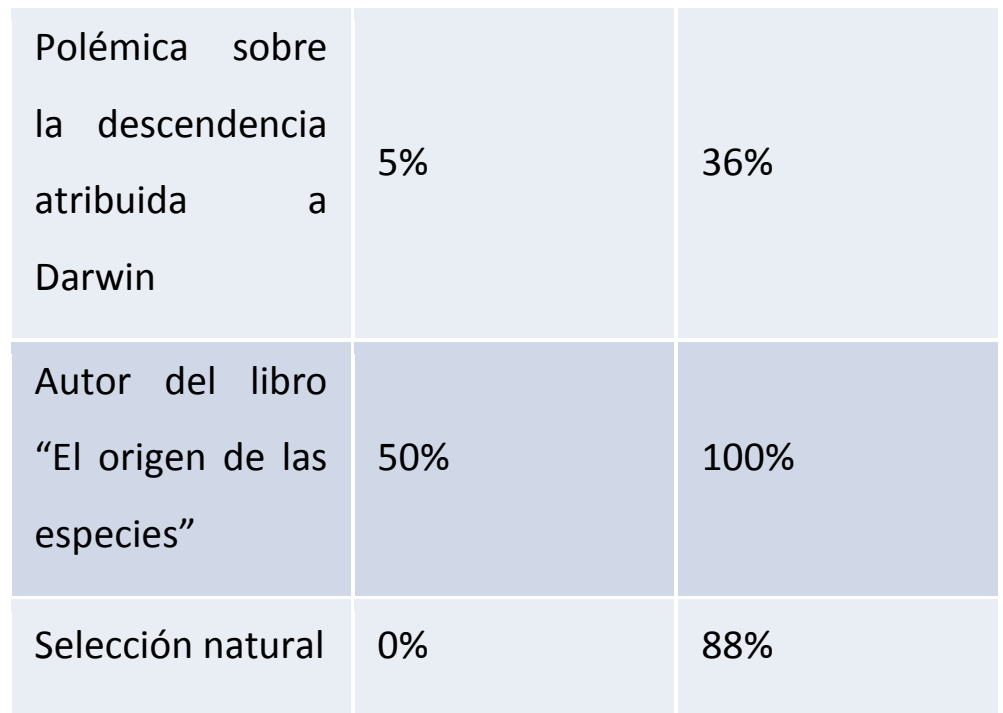

En la siguiente tabla se observa que hay un aumento en las respuestas correctas del grupo control, en donde el dato más significativo es el porcentaje para el concepto de selección natural con un $88 \%$ de incremento después de la intervención pedagógica.

\section{Comparación del pretest y postest en el grupo experimental.}

En el grupo experimental los alumnos mostraron un aumento en sus porcentajes de respuestas correctas para el concepto de cambio gradual fue de un 24-61\%, para el concepto de caracteres adquiridos fue de un 16-61\%. En cuanto a la idea errónea de fue Darwin el que mencionó la descendencia del mono paso de 0-17\%, para el dato del autor del libro "El origen de las especies" paso de $72-100 \%$. Para el concepto de selección natural el grupo mostró un aumento del $8 \%$ al $74 \%$ de respuestas correctas. Con respecto a la contribución de Wallace el $74 \%$ respondió correctamente a diferencia del $16 \%$ inicial. Sin embargo en la pregunta 9, donde se les pregunta acerca del esquema que sigue la teoría de Lamarck, se confunden entre cambio gradual y la herencia de las capacidades.

Como se puede observar hubo un aumento el porcentaje de respuestas correctas después del uso de la narrativa, coincidiendo con las investigaciones de Solbes y Traver (2001) y Kalkanis y colaboradores (2010), quienes afirman que es posible introducir aspectos de historia en los materiales didácticos elaborados para la enseñanza, pues se detecta una mejor comprensión de los aspectos relacionados con la contextualización de los conocimientos científico.

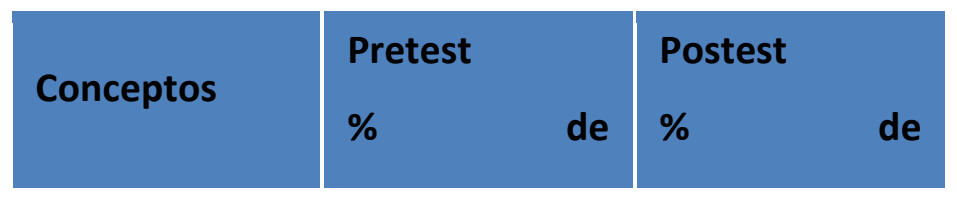




\begin{tabular}{|l|l|l|}
\hline & $\begin{array}{l}\text { respuestas } \\
\text { correctas }\end{array}$ & $\begin{array}{l}\text { respuestas } \\
\text { correctas }\end{array}$ \\
\hline $\begin{array}{l}\text { Cambio } \\
\text { gradual }\end{array}$ & $61 \%$ \\
\hline $\begin{array}{l}\text { Herencia de los } \\
\text { caracteres } \\
\text { adquiridos }\end{array}$ & $16 \%$ & $61 \%$ \\
\hline $\begin{array}{l}\text { Polémica sobre } \\
\text { la }\end{array}$ & & \\
\hline descendencia \\
atribuida
\end{tabular}

\section{Conclusión}

Se puede concluir que:

- Los alumnos de educación media superior presentan una preconcepción lamarckista del proceso evolutivo, en donde prevalecen las ideas de que los organismos cambian por necesidad de adaptarse al medio y que dichos cambios son heredables.

- A pesar de las críticas a las cuales puede ser sujeto el uso de cuestionarios para evaluar sólo contenidos declarativos y no procesos, los resultados obtenidos en esta investigación se suman al objetivo de encontrar esquemas causales y principios comunes sobre las ideas previas de los alumnos para ofrecer 
a los docentes información importante al momento de programar estrategias de enseñanzaaprendizaje.

- La narrativa posee eficacia como estrategia didáctica para la enseñanza de contenidos científicos.

- Los conceptos en donde se observó mejoría en los alumnos fueron:

$\sim$ Cambio gradual

Herencia de los caracteres adquiridos

Selección Natural

- Para datos históricos

Mito sobre Darwin y la descendencia del mono.

Autor del libro "El origen de las especies"

Finalmente este trabajo partió del interés de mejorar la enseñanza de la teoría evolutiva en el bachillerato, ya que como se pudo observar los alumnos siguen manteniendo una interpretación lamarckiana y desconocen aspectos históricos del proceso científico.

Aportar evidencias para los profesores de que necesitan actualización sobre los temas a enseñar, pues en esta investigación algunos de ellos siguen compartiendo las mismas ideas que sus alumnos, con el objetivo de desarrollar metodologías acordes a las necesidades de su contexto educativo, pues la intervención del profesor es fundamental en el proceso de enseñanza aprendizaje y que esta mediación pedagógica se puede apoyar en una serie de herramientas didácticas, elaboradas por el mismo o elegir las adecuadas en una planeación meticulosa.

\section{Bibliografía}

Bishop, B.A. \& Anderson C.W. (1990). Student conceptions of natural selection and its role in evolution. Journal of Research in Sciencie Teaching, 27 (5), 415-427.

Campanario, J.M. (1998). La ciencia que no enseñamos. Enseñanza de las Ciencias, 17 (3), 397-410.

Chinn, C. A., \& W. F. Brewer. (1993). The role of anomalous data in knowledge acquisition: A theoretical framework and implications for instruction. Rev. Ed. Res. 63 (1), 1-49. 
Díaz-Barriga F., Hernández, G. (2006). Estrategias docentes para un aprendizaje significativo. Una interpretación constructivista. México: Editorial McGraw Hill.

Jiménez-Aleixandre, M. P. (1992). Thinking about theories or thinking with theories? A classroom study with Natural Selection. International Journal of Sciencie Education (14), 51-61.

Kalkanis, A., García, J. \& Rodríguez, D. (2010). Influencia de los cuentos como recurso didáctico en el proceso de Enseñanza-Aprendizaje para la comprensión y aplicación de la tabla periódica en la Química de noveno grado de educación básica. Revista Ciencias de la Educación., 20 (35), 110-132.

Lawson, A.E. \& Worsnop, W. A. (1992). Learning about evolution and rejecting a belief in special creation. J. Res. Sci. Teach. 29(2), 143-166.

Singer, T. (2006). Efecto del cuento como estrategia de enseñanza y aprendizaje sobre el rendimiento estudiantil en el contenido de los compuestos químicos inorgánicos. Tesis, Universidad Pedagógica Experimental Libertador. Barquisimeto Estado de Lara. Venezuela

Solbes, J. \& Traver, M. 2001. Resultados obtenidos introduciendo historia de la ciencia en las clases de Física y Química: mejora la imagen de la ciencia y desarrollo de actitudes positivas. Enseñanza de las Ciencias, 19(1), 151-162. 